\section{Hubble telescope delays prove a boon for some}

\section{Berkeley}

Although frustrating, the three-year delay in launching the Hubble Space Telescope has proved a boon for at least one of the mission's scientific teams. During ground testing of the faint object spectrograph (FOS), project manager Larry Randall says "fundamental problems" were found that were causing $10-30$ per cent reductions in the light-gathering capability of the blue-side and red-side spectrographs. Tests of the instrument's optical alignment revealed a 'cat's eye' where there should have been an unbroken disk of light.

Randall received permission to open the instrument in late 1986. Inside, he discovered insulation and baffle material hanging in the light beam. And pieces of black tape and a rubber glove were loose within the instrument where they could stray into the light path in a microgravity environment. Randall also found that the instrument did not have the proper optical alignment, a problem he thinks may have been caused by the unfamiliarity of the instrument's contractor - Martin Marietta - with proper alignment techniques. Lou Ripp of Martin Marietta who was involved with the FOS project, said through a spokesman that the company had made a mistake in not involving scientists more actively in the design from the start.

In the overhaul, the FOS light path was restored and the opportunity taken to fit a new detector for the red-side spectrograph. The detector uses recently declassified technology developed by a project member, Edward Beaver of the University of California, San Diego, that has been used in military night vision devices.

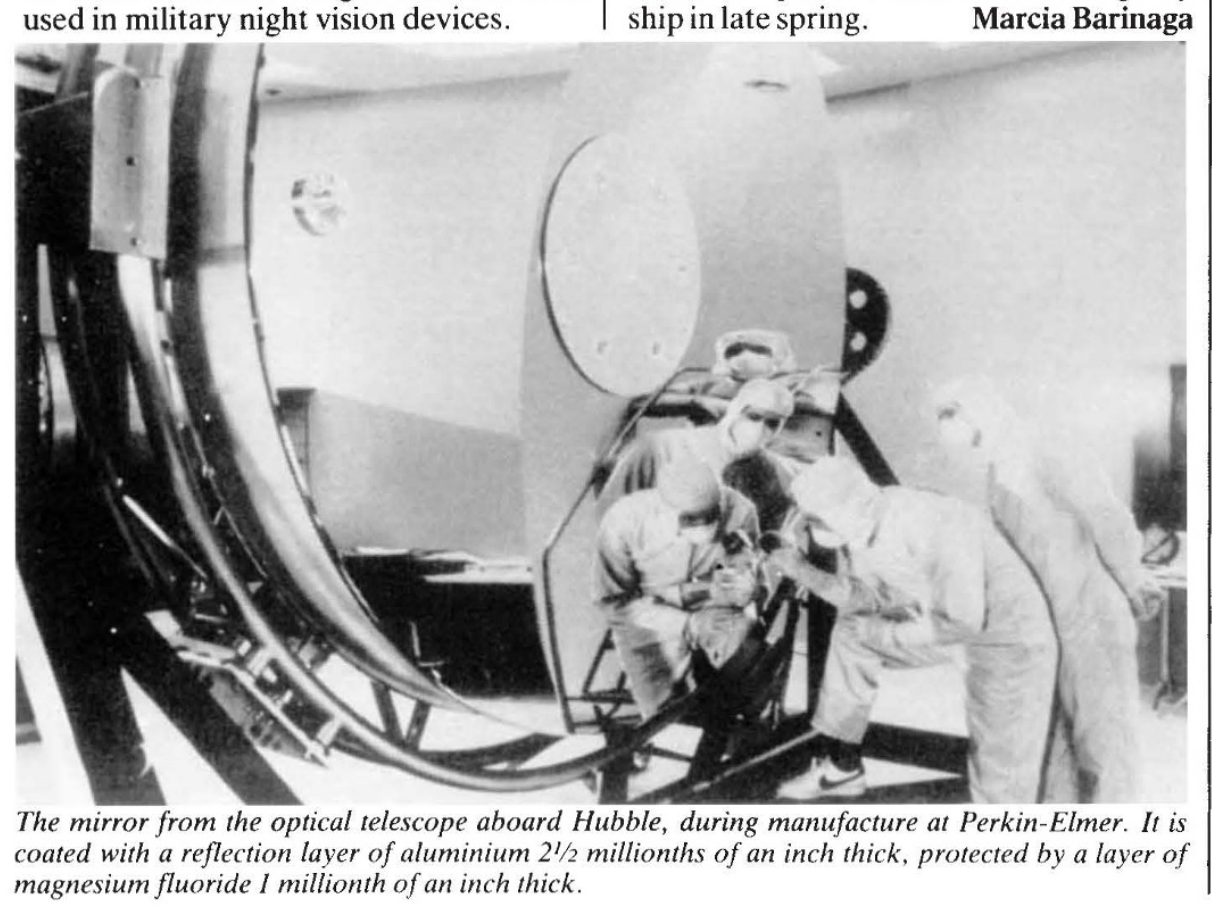

The mirror from the optical telescope aboard Hubble, during manufacture at Perkin-Elmer. It is coated with a reflection layer of aluminium $2^{1 / 2}$ millionths of an inch thick, protected by a layer of magnesium fluoride I millionth of an inch thick.

The Hubble Space Telescope is now in Sunnyvale, California, where it is in the final stages of ground testing for its December launch. Apart from the changes made to the FOS, the alterations of the telescope's other four instruments were limited to refurbishing of parts to lengthen the lifetime of instruments, and the replacement of a faulty power supply on the high speed photometer, according to Fred Wojtalik, project deputy manager.

The telescope will soon receive its new improved solar wings from the European Space Agency, modified not only to increase power levels by 15 per cent, but also to resist degradation by atomic oxygen encountered in low Earth orbit. The newly developed nickel-hydrogen batteries ( Nature 326, 630; 1987) are still in final testing, but if they do not prove suitable Wojtalik says the craft can operate with the batteries from the original design.

The last remaining major decision is whether the telescope will travel to Florida by air or sea. NASA (National Aeronautics and Space Administration) originally planned to ship the craft through the Panama Canal on an icebreaker. But, because the ship's busy schedule would allow only a narrow time window for the trip, mission planners proposed to fly the telescope to Florida on a C-5A transport, in an Air Force container

The telescope will fly to its Florida launch pad in August unless next month's flight tests show that the Air Force container, which was built for other purposes, is unable adequately to support and protect the telescope, in which case it will go by ship in late spring. Marcia Barinaga
UK plans for astronomy and planetary science London

A PLAN for Britain's research in groundbased astronomy and planetary science over the next 20 years is published this week by the Science and Engineering Council. It focuses on four projects. The main one is an extension of the MERLIN radio-interferometer, based at the Nuffield Radio Astronomy Laboratories at Jodrell Bank, by the construction of a new 32metre radio-telescope at Cambridge. This will also enhance British participation in the European Very-Long-Baseline Interferometry Network; construction of the telescope, to begin this year, will cost £6 million.

The council also recommends that Britain seeks a 25 per cent share, at a cost of $£ 2$ million, in a new incoherent scatter radar at Spitzbergen in the Arctic and at least a 30 per cent share in a new 1-km gravitational radiation observatory. The council is seeking extra money from the Advisory Board for the Research Councils so that Britain can afford a greater share in the observatory and thus secure its siting in Scotland.

The fourth project in the plan is the construction of an 8-metre telescope optimized for both optical and infrared work. At a cost of $£ 15$ million over the next ten years, Britain would hope to have a 50 per cent share in the telescope.

The plan also identifies 17 smaller projects which may be selected according to available resources. $£ 28$ million is available for the programme until the year 2000 , but the board says that development of the new facilities will require savings in existing ones; these will be made through creating more international partnerships, reducing operating costs and closing some facilities. It does not specify where the cuts might be made.

Christine McGourty

\section{Israeli satellite stars}

\section{Jerusalem}

OFEQ 1, Israel's first satellite, burnt up after re-entering the Earth's atmosphere (over the Pacific Ocean) on 14 January, 118 days after launch. The 156-kg experimental communications satellite was expected to remain in orbit for only six weeks. Professor Yuval Ne'eman, head of the Israeli space agency, attributed the extended life span to the fact that Ofeq 1 (Horizon 1) reached "unexpected" altitudes, thereby encountering fewer particles in space. He said the satellite continued to transmit data on the Earth's environment and magnetic field throughout its orbit.

Ofeq II, a more sophisticated satellite due to carry a scientific payload, is being prepared for launch within two years. With Ofeq 1 , Israel became the ninth country to launch a satellite. 